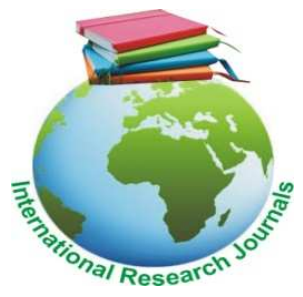

Journal of Medicine and Medical Sciences Vol. 8(5) pp. 054-059, August 2017

DOI: http:/dx.doi.org/10.14303/jmms.2017.050

Available online http://www.interesjournals.org/JMMS

Copyright (C) 2017 International Research Journals

Full Length Research Paper

\title{
Guided implant placement using the trephine drill non- sleeve and immediate provisional crown or bridge in the esthetic zone
}

\author{
Suchat $K^{1}$, Wipasara $N^{2}$, Thipsuda $N^{1}$, Sunithi $T^{1}$, Siriwan $T^{1}$, Arunsri $M^{1}$, Siriluk $\mathbf{P}^{1}$, Puntanim \\ $K^{1}$, Thirachai $S^{1}$, Chaweewan $P^{2}$, Lertrit $S^{3}$, Wirun $\mathrm{T}^{4}$, Kriskrai $\mathrm{S}^{4}$, *Nawakamon $\mathrm{S}^{1}$ \\ ${ }^{1}$ Prachatipat Hospital, Institute of dentistry, Department of Medical Services \\ ${ }^{2}$ Ladlumkeaw Hospital, Institute of dentistry, Department of Medical Services \\ ${ }^{3}$ Ministry of Public Health, Thammasat University \\ ${ }^{4}$ National Metal and Materials Technology Center \\ *Corresponding Author's Email: nawakamons@hotmail.com
}

\begin{abstract}
The immediate restoration of dental implants has shown revealed the clinical success rates compare with conventional multi-step protocol. However, the accuracy of guided dental implant placement using guided trephine drill and the restoration affect the treatment success and more security. To provide the professional tool improve the planning to choose the implant size and position. The objectives of this study were to evaluate the accuracy and position of the digital surgical guide before and after implant placement with the guided trephine drill. The digital process chain work from Intra oral scan with denture will be performed. And then cone beam computer tomography (CBCT) with gutta-percha marker denture. The virtual prototyping precisions of 5 stereolithographic surgical splints are validate a design before committing to making the rapid prototype were excellent. The satisfaction score compare with the conventional splint and provisional crown improve in term of time and esthetic outcome. The guided trephine drill can reduce the stage of bone augmentation. The mean measurement differences in accuracy between the computer-planned implant and the before and after surgery for 6 implants guided placed were $1.18 \mathrm{~mm}$ for the apex (SD+/- 0.37), $0.35 \mathrm{~mm}$ for the mesial $(\mathrm{SD}+/-1.2) 0.35 \mathrm{~mm}(\mathrm{SD}+/-1.5)$, for the depth deviation. The results show a statistically significant difference of the implant placed between the conventional and the virtually planed implant positions with the CAD/CAM-guided surgical template.
\end{abstract}

Keywords: CBCT, Non-sleeve surgical guide, Trephine, Guided surgery, Dental implant

\section{INTRODUCTION}

Currently surgical guided provisional prosthesis is used to provide safe treatment demonstrates and long term success in the esthetics zone.

The software starts by placing virtual crowns according to prosthodontics setting (Flugge et al., 2016). The implant is designed to support crown or bridge by using the 3Shape software. The plan was to fabricate CAD/CAM zirconia anatomically abutment crown and bridge. From CBCT we measure house field unit at the osteotomy site to confirm bone quality. The CBCT images in this study demonstrated an average thickness 1-2 mm. of the facial bone wall at various levels.
Preparation of the implant bed with sequences of bone drill. It was planned to connect a definitive prefabricated abutment and a provisional restoration to the implant immediately after surgery (Marchack, 2007). Immediate restoration loading can be placed when the implant achieved good primary stability of adequate length ( $\geq 8$ $\mathrm{mm}$ ) and diameter $(\geq 4 \mathrm{~mm})$. The restoration should not be removed during the healing period of approximately 6 weeks. The patient is instructed of how to function and take care of adequate oral hygiene during the healing period. Screw-retained provisional restorations are recommended. Immediate restoration and loading can be 
used when the bone volume at the site was close to ideal no simultaneous guided bone regeneration procedures are required. Patient satisfaction is the most crucial important factor in predicting the success of implant therapy especially at the anterior maxilla.

Implant placement has become a routine modality to replace the missing teeth. Recently, computer-aided design and manufacture have made it possible. To use data from computerized tomography to plan prosthesis restoration, but also apply and transfer this information to the surgery (Tahmaseb et al., 2009). The prediction and success of aesthetic implant treatment is depends on an accurate diagnosis and development of a restorative driven the surgical treatment plan (Holst et al., 2007). The use of technology such as CBCT guided surgery, growth factors may enhance the outcomes. The software and hardware advances are substantially changing the way dental restorations. Digital workflow provides a competitive treatment options to provide dental professionals with the most efficient and comprehensive solutions (Ritter et al., 2009).

Conventional procedure for dental implant placement begins with a small drilling site to prepare a first hole. Then the first hole is progressively enlarged in order to fit properly with a dental implant having a standard diameter 2-6 $\mathrm{mm}$ approximately. In most cases, a bone grafting is also required. Therefore, by the conventional procedure, a synthetic bone is needed to use with an implant. An attempt has been made to develop a drilling device which can be preserved an autologous bone to use for grafting with dental implant procedure. Affixing a ring to a surgical template for preparing a ring-affixed template on a predetermined implant site; aligning a cutting end of dental drilling device within the ring; and cutting bone in corresponding with an implant size.

Dental Implants are one of the most significant advancements in dentistry in the past two decades. Today, the dental implant is becoming the standard of care to replace missing teeth. The advancements in the science and engineering of dental implants add a whole new dimension to the replacement of the missing tooth. The evidence supports the immediate restoration of dental implants reports the clinical success rates compare with conventional multi-step protocol. This study aims to evaluate the predictability and success of aesthetic guided implant placement using guided trephine drill and immediate provisional crown or bridge in esthetic zone. The main objective of this study is to evaluate the accuracy of the digital surgical guide using the guided trephine drill and the position of the implant placement with the conventional placement. The surgical technique and the loading conditions influence the stability of the implants and the success of osseointegration. Through advanced digital engineering, dentists are now able to offer precise guided planning, digital impressions, CAD/CAM models, and restorations. These techniques are providing the revolutionary opportunity for the dental team to collaborate and plan dental implant cases virtually with more effectively and longtime esthetic restorations (Pettersson et al., 2010).

Dental implant systems have improved their interface attachments at the mucosal surface level of the implant which reduces abutment loosening and enhances overall esthetics. However, while dental implant technology has significantly advanced in the past twenty five years from both a surgical and restorative standpoint, there have been some limitations. These limitations in many ways have limited the number of dentists that offer dental implants to their patients. While dental implants have seen significant advancements, these precision designed and engineered fixtures have relied on traditional nonprecision manual surgical placement, traditional restorative methods, and traditional laboratory techniques for the delivery and replacement of the missing tooth.

\section{MATERIALS AND METHODS}

The digital process chain work from Intra oral scan (Trios 3 Shape) with and without try in denture will be performed and then CBCT (Newton Accord.,co.th) without denture. Then crop CBCT in 3 dimensions later superimpose the images later in the software define panoramic curve. The software starts by placing virtual crowns according to prosthodontic setting. Proper depth and angulation were determined, ensuring that the implants were positioned to accommodate a maximum amount of bone while establishing optimal support and the platform needed to achieve an esthetic outcome for the proposed restoration. Ethic approve No. PPHO-REC 2559/001

\section{Intraoral scanning and CAD/CAM in dentistry}

One of the critical components of the digital implant dentistry workflow involves the digital capture of the patient's dentition for planning, placement, and restoration. Intraoral scanning in its simplest form is a way for the dental team to take an impression without traditional impression material and create an accurate virtual model of the patient's dentition. Predictable cost and more predictable outcomes. Once the tooth is scanned and the structured data is captured, software is then used to process each data point to create a geometrical virtual three-dimensional model. This virtual model now can be used by the dentist or lab technician in a variety of applications. The viewing software can allow the dental team to virtually share the models digitally for treatment planning and discussion.

Additionally, the dental team can utilize the models to process virtual abutments, copings, crowns, removable prosthesis and other orthotic devices without a physical model through computer aided design (CAD) software applications. Once the CAD software applications have 
056 J. Med. Med. Sci.

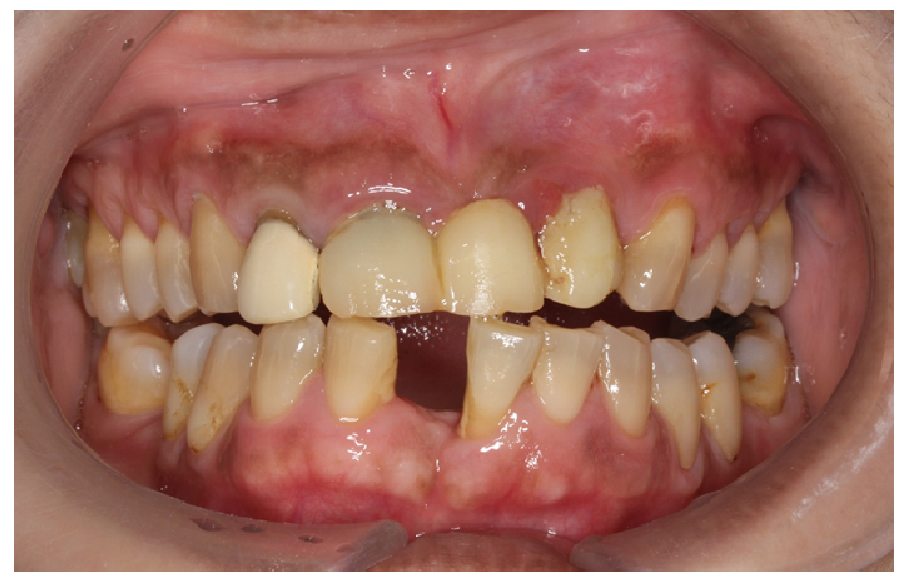

Figure1: Patient age 53 years coming with unpleasant with upper front teeth from 12-22 after discussion with patients, all problematic restored teeth was planned to remove.

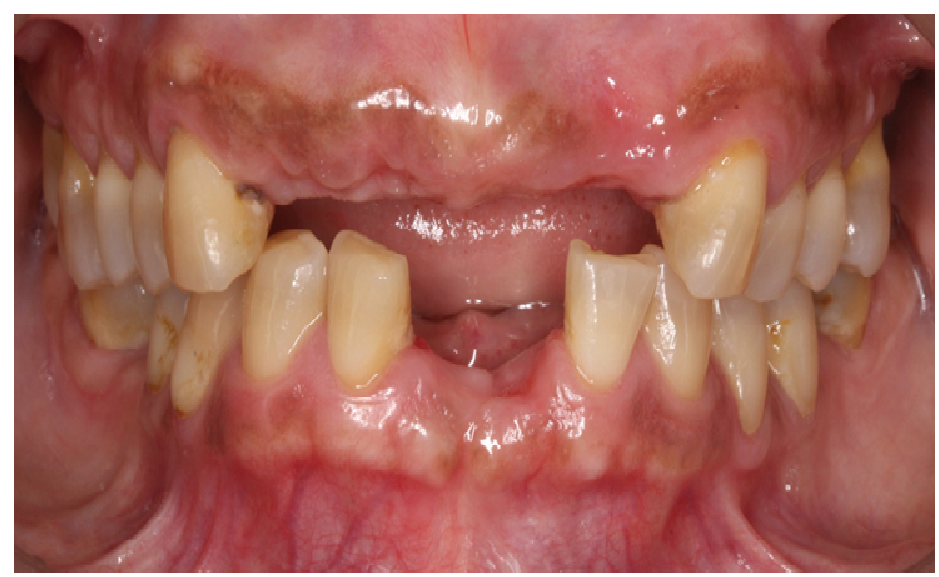

Figure 2: After 2 week remove the teeth.

created the virtual geometric three-dimensional model, computer aided manufacturing (CAM) techniques use various printing and milling machines to create an exact replica of the virtual model in a physical form (Proussaefs, 2016). It is through this process that the dental team can utilize the scanning technology to create more accurate models, abutments, and restorations for the dental implant patient.

\section{Digital dental implant scanning}

Obtaining an accurate impression to isolate the position of the implant in the patient's mouth is critical to providing the precision needed for the dental restoration. Traditionally, dental implant impression techniques utilize impression copings that are inserted into the implant at the time of impression. Traditional impression materials such as polyvinyl siloxane or polyether are used to capture the representative teeth and mucosa in the impression tray. Once the impression is taken, the implant analogs are attached to the impression copings and a stone model is created with the analogs representing the positioning of the implant in the model. While this technique has been used in implant dentistry for several years, there are several layers of potential inaccuracies associated with the various steps involved.

In order to minimize these steps and streamline the workflow of the implant impression, a digital scan body was created. The scan body replaces the traditional impression coping and allows the implant fixture to be captured with an intraoral digital scanning on device. The scan bodies fit precisely into the dental implant fixture in the mouth to allow for accurate capture of the associated 


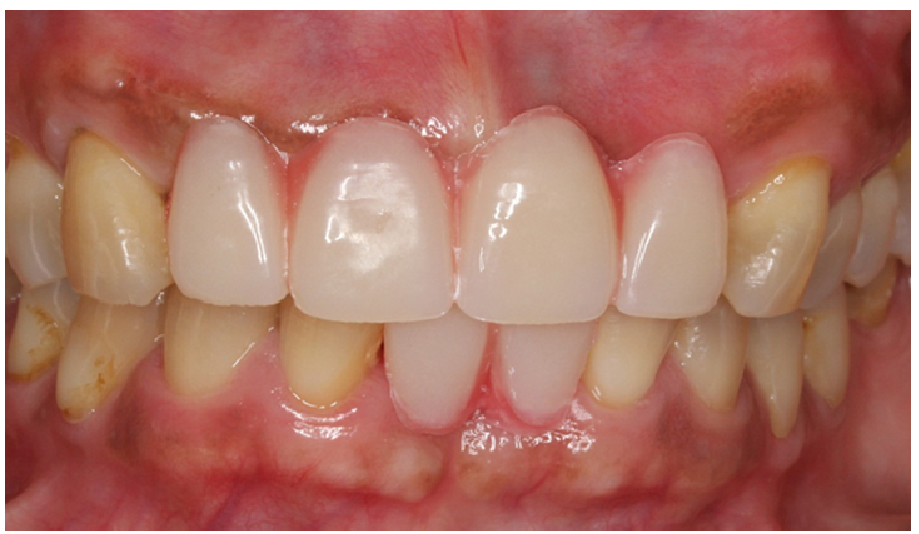

Figure 3: The provisional was tried in to make all the information's to patient and dental technician (Pre-preparation scan)

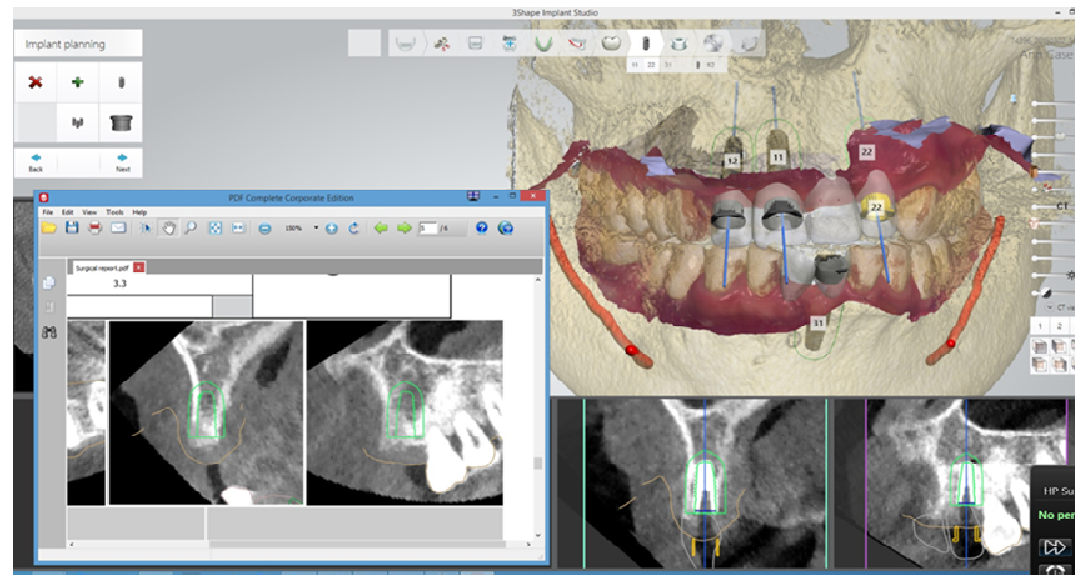

Figure 4: The Merge intraoral scan and CBCT for planning according to the final restoration then take CBCT after implant were placed.
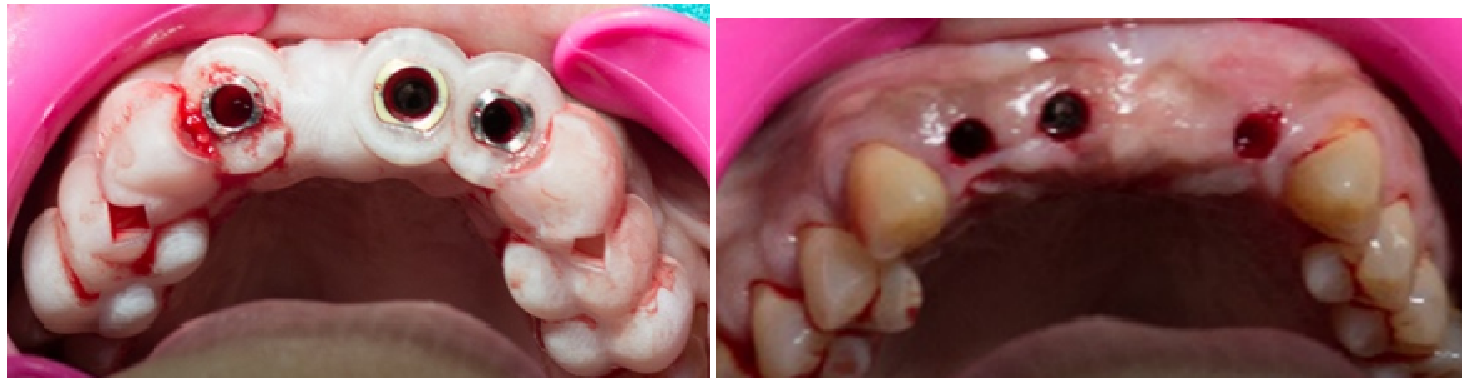

Figure 5 and 6: Design drill guide and do flapless operation up to the amount of the bone and soft tissue.

teeth and mucosa in reference to the position of the implant fixture, just as a traditional implant impression coping end. However, the scan body has a precise geometrical shape on the surface to allow for optical capture of the fixture. Once the scan body image is captured and registered the CAD software, through 
058 J. Med. Med. Sci.



Figure 7: Guided trephine use as the guided drill provide the amount of bone block that will custom for make contour around implant placement.
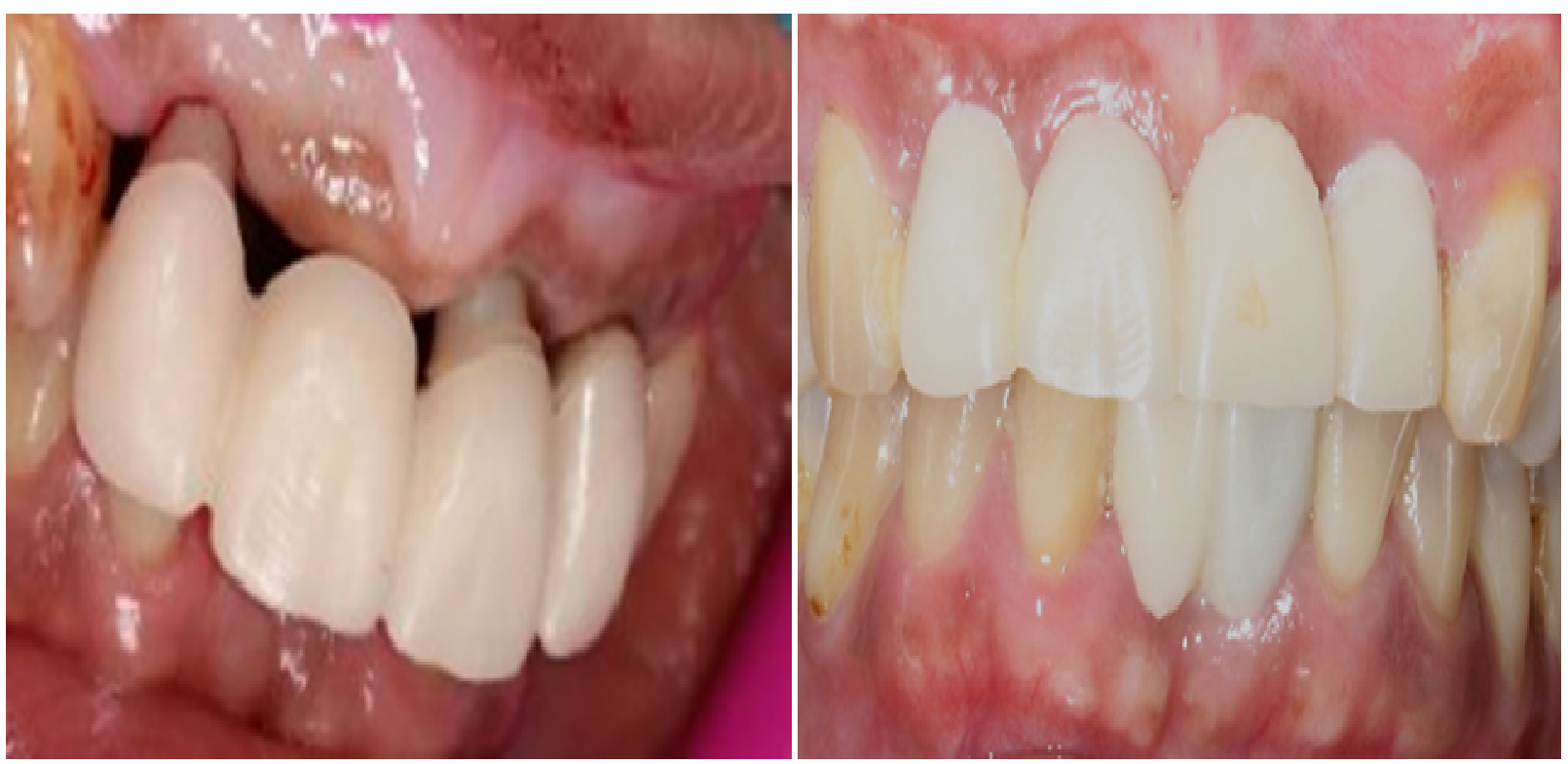

Figure 8 and 9: Immediate placement CAD/CAM provisional crown and bridge at the day of surgery.

alignment algorithms, can accurately position the implant into the virtual model. These studies describe the implant placement by using the guided trephine drill and the digital workflow with CAD/CAM technology (Tahmaseb et al., 2009).

After the drill guide is designed, then we import the file of implant position are imported as a file to design as cement retain or screw retain abutment on titanium base abutment then design the occlusion. In case we need the digital analog we can print digital model to try in. In one visit or one day case will choice the restoration block that will be milling for the temporary or final restoration. 


\section{RESULTS}

The results of the study are shown in the table below:

Report

\begin{tabular}{|c|c|c|c|c|c|}
\hline \multicolumn{2}{|c|}{ Tooth } & \multirow{2}{*}{$\begin{array}{c}\text { Vertical } \\
.63\end{array}$} & \multirow{2}{*}{$\begin{array}{c}\text { Mesial } \\
.27\end{array}$} & \multirow{2}{*}{$\begin{array}{c}\text { Distal } \\
.36\end{array}$} & \multirow{2}{*}{$\begin{array}{c}\text { Angulation } \\
11.80\end{array}$} \\
\hline 11 & Mean & & & & \\
\hline & $\mathrm{N}$ & 2 & 2 & 2 & 2 \\
\hline & Std. Deviation & .19 & .07 & .03 & .84 \\
\hline \multirow[t]{3}{*}{12} & Mean & .97 & .41 & .43 & 8.37 \\
\hline & $\mathrm{N}$ & 2 & 2 & 2 & 2 \\
\hline & Std. Deviation & .14 & .18 & .27 & .08 \\
\hline \multirow[t]{3}{*}{13} & Mean & 1.30 & .21 & .29 & 22.37 \\
\hline & $\mathrm{N}$ & 2 & 2 & 2 & 2 \\
\hline & Std. Deviation & .03 & .01 & .05 & .07 \\
\hline \multirow[t]{3}{*}{21} & Mean & .98 & .43 & .31 & 10.29 \\
\hline & $\mathrm{N}$ & 2 & 2 & 2 & 2 \\
\hline & Std. Deviation & .48 & .13 & .04 & 4.81 \\
\hline \multirow[t]{3}{*}{22} & Mean & .98 & .36 & .23 & 16.57 \\
\hline & $\mathrm{N}$ & 1 & 1 & 1 & 1 \\
\hline & Std. Deviation & . & . & . & . \\
\hline \multirow[t]{3}{*}{23} & Mean & 1.74 & .43 & .65 & 24.44 \\
\hline & $\mathrm{N}$ & 1 & 1 & 1 & 1 \\
\hline & Std. Deviation & . & . & . & . \\
\hline \multirow[t]{3}{*}{ Total } & Mean & 1.18 & .35 & .37 & 14.67 \\
\hline & $\mathrm{N}$ & 10 & 10 & 10 & 10 \\
\hline & Std. Deviation & .37 & .12 & .15 & 6.46 \\
\hline
\end{tabular}

\section{DISCUSSION}

Dental implant companies are now taking advantage of the new digital solutions in dentistry to improve dental implant ease of use, and provide more precision and cost effective delivery (Noh et al., 2016). Precision surgical guided placement using restorative driven threedimensional imaging and planning allows for more predictable surgical placement. High surface digital impression scanning and CAD/CAM milling allows for precision design and modeling for improved fit and accuracy of both the abutment and restorations (Marchack, 2007). Combining these digital technologies allows the dentist to plan and treat the patient in a more efficient manner with more predictable results. The digital implant dentistry workflow is now redefining and paving a new standard of delivery for the replacement of missing teeth, giving the dental team more confidence when treating their patients.

\section{REFERENCES}

Choi W, Nguyen BC, Doan A, Girod S, Gaudilliere B, Gaudilliere D. Freehand Versus Guided Surgery: Factors Influencing Accuracy of Dental Implant Placement. Implant dentistry. 2017.

Holst S, Blatz MB, Eitner S (2007). Precision for computer-guided implant placement: using $3 D$ planning software and fixed intraoral reference points. J Oral Maxillofac Surg. 65(3): p. 393-9.

Marchack CB (2007). CAD/CAM-guided implant surgery and fabrication of an immediately loaded prosthesis for a partially edentulous patient. J Prosthet Dent. 97(6): p. 389-94.

Noh K, Ahran P,Jung-WL,Yong-D (2016). Fabricating a tooth- and implant-supported maxillary obturator for a patient after maxillectomy with computer-guided surgery and CAD/CAM technology: A clinical report. J Prosthet Dent. 115(5): p. 637-42.

Pettersson A, Kero T, Gillot L, Cannas B, Fäldt J, Söderberg R, Näsström K (2010). Accuracy of CAD/CAM-guided surgical template implant surgery on human cadavers: Part I. J Prosthet Dent. 103(6): p. 334-42.

Proussaefs P (2016). Use of CAD/CAM Healing Abutment Immediately After Dental Implant Placement for the Non-Esthetic Zone: A Guided Soft Tissue Healing Technique. J Oral Implantol. 42(2): p. 189-93.

Ritter L, Neugebauer J, Dreiseidler T, Rothamel D, Cizek J, Karapetian VE, Mischkowski RA, Bindl A, Zöller JE (2009). 3D X-ray meets CAD/CAM dentistry: a novel procedure for virtual dental implant planning. Int J Comput Dent. 12(1): p. 29-40.

Tabea F,Wiebe D,Jobine P,Bassam H,Katja N,Daniel W (2016). Registration of cone beam computed tomography data and intraoral surface scans - A prerequisite for guided implant surgery with CAD/CAM drilling guides. Clin Oral Implants Res.

Tahmaseb A, De Clerck R, Wismeijer D (2009). Computer-guided implant placement: $3 D$ planning software, fixed intraoral reference points, and CAD/CAM technology. A case report. Int J Oral Maxillofac Implants. 24(3): p. 541-6. 